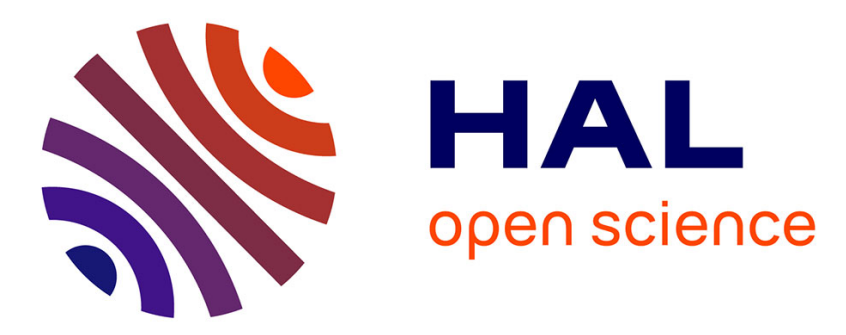

\title{
Les parties du discours dans la grammaire sanskrite de tradition pāṇinéenne
}

Emilie Aussant

\section{To cite this version:}

Emilie Aussant. Les parties du discours dans la grammaire sanskrite de tradition pāṇinéenne. Bernard Colombat; Aimée Lahaussois. Histoire des parties du discours, Peeters, p. 491-505, 2019, 978-90-4293952-3. halshs-02305968

\section{HAL Id: halshs-02305968 \\ https://shs.hal.science/halshs-02305968}

Submitted on 24 Mar 2020

HAL is a multi-disciplinary open access archive for the deposit and dissemination of scientific research documents, whether they are published or not. The documents may come from teaching and research institutions in France or abroad, or from public or private research centers.
L'archive ouverte pluridisciplinaire HAL, est destinée au dépôt et à la diffusion de documents scientifiques de niveau recherche, publiés ou non, émanant des établissements d'enseignement et de recherche français ou étrangers, des laboratoires publics ou privés. 


\section{Les parties du discours dans la grammaire sanskrite de tradition pāṇinéenne}

\section{Présentation}

La tradition grammaticale sanskrite est la plus ancienne (son texte fondateur aurait été composé aux alentours du $\mathrm{V}^{\mathrm{e}} \mathrm{s}$. avant notre ère) et la plus longue qui soit (on considère traditionnellement qu'elle s'achève au XVIII ${ }^{\mathrm{e}}$ s., avec l'œuvre de Nāgeśa Bhaț̣a). La discipline grammaticale (en sanskrit, vyākaranal ${ }^{1}$ ) constitue l'une des six disciplines d'érudition (vedāng $a^{2}$ ) associées aux Veda ${ }^{3}$. Le plus ancien texte de Vyākaraṇa qui nous soit parvenu est l'Asțāadhyāyā (ci-après A) de Pāninini ${ }^{4}$. Cet ouvrage servit de base à l'établissement d'une école de grammaire qui, tout au moins d'après ce que nous pouvons déduire des textes qui nous sont parvenus, domina largement jusqu'au $\mathrm{XVIII}^{\mathrm{e}} \mathrm{s}$. Comme pour d'autres disciplines de l'Inde ancienne, le succès - spontané ou organisé - du texte fondateur du Vyākaraṇa fit qu'il éclipsa durablement les ouvrages issus d'autres écoles.

La plus ancienne trace que nous ayons d'une segmentation de la chaîne parlée dans l'Inde ancienne est liée à la transmission des Veda et, plus précisément, aux techniques de récitation de ces textes (ce point est détaillé ci-après, dans la section pada). Les unités délimitées dans ce cadre, les pada («mot»), ont fait, plus tard, l'objet de deux classifications principales au sein de la discipline grammaticale, l'une sémantique, l'autre formelle. On trouve mention de la première, qui passe pour (et qui semble bien) être la plus ancienne, dans le Nirukta de Yāska (composé entre le VII et le $\mathrm{III}^{\mathrm{e}}$ s. avant notre ère), le seul traité d'explication sémantique qui nous soit parvenu. Les pada y sont classés en quatre catégories : näman («nom ») et ākhyāta («verbe »), upasarga («préposition ») et nipāta («particule»). Les noms sont présentés comme référant à une substance (sattva) et les verbes, à un processus $(b h \bar{a} v a)$; les prépositions font l'objet de deux interprétations: elles sont, pour certains auteurs, indicatrices (dyotaka) d'un sens alors que pour d'autres, elles expriment directement (vācaka) un sens ; quant aux particules, elles sont présentées comme exprimant des sens variés ${ }^{5}$. Cette classification des pada en quatre catégories se retrouve fréquemment dans les textes grammaticaux (et même au-delà ${ }^{6}$ ), à l'exception notable de l'Aștâadhyāyà de Pāṇini.

\footnotetext{
1 Le terme vyākaraṇa est un nom d'action dérivé de la racine préverbée $v y-\bar{a}-k r$-. On l'interprète traditionnellement comme dénotant un processus de séparation ou de discrimination des constituants, mais on peut aussi y voir une référence à un processus de création, qui génère, de façon diversifiée, les unités linguistiques (cf. Thieme 1982-1983, p. 11 et 23-34 ; Cardona 1997a, p. 565-571).

2 Litt. «auxiliaire des Veda». Trois des cinq autres Vedānga concernent le langage : la phonétique (śikșā, «enseignement»), la métrique (chandas, «texte versifié, mètre ») et l'explication sémantique (nirvacana, « explication »).

${ }^{3}$ Les Veda constituent le corpus fondateur de la religion védique (la forme la plus ancienne du brahmanisme), point de départ de la plupart des doctrines de l'Inde ancienne. Ces textes, dont le plus ancien remonte à la seconde moitié du second millénaire avant notre ère, sont fondés sur une « révélation » (śruti, littéralement « audition»). Les Veda se composent de samhitā, collections versifiées d'hymnes, de prières, d'incantations rituelles et de commentaires des saṃhitāa. Il y a quatre types de saṃhitā : 1) les strophes (rcc), qui sont récitées au cours des cérémonies rituelles et qui composent la Rksaṃhitā ou le Rgveda, 2) les incantations rituelles (yajus), rassemblées (avec ou sans commentaires) dans la Yajuhsamhitā ou le Yajurveda, 3) les chants (sāman) de la Sāmasamhitā ou du Sāmaveda et 4) les formules (atharvan) de l'Atharvasaṃitā ou de l'Atharvaveda.

4 Pānini mentionne néanmoins le nom de dix prédécesseurs.

${ }^{5}$ La distinction entre noms et verbes d'un côté, prépositions et particules de l'autre, est clairement marquée dans la formule sanskrite qui énumère les quatre classes (nāma-ākhyāte ca_upasarga-nipātaś ca). Cette distinction peut s'expliquer de deux manières : 1) les deux groupes d'unités se différencient par le type de référent auquel elles renvoient (une chose qui peut être pointée $v s$ une chose qui ne peut pas l'être) ; 2) les unités se différencient par la manière dont elles renvoient à leur référent (de manière indépendante $v s$ de manière dépendante).

${ }^{6}$ On la trouve notamment dans un traité de dramaturgie et dans un traité de politique.
} 
La seconde grande classification des pada, fondée sur un critère purement formel, se trouve justement dans le traité grammatical composé par Pānini ${ }^{7}$. Le grammairien y distingue deux grands types de pada, les $s U B$-anta «noms » (litt. «ce qui se termine par une désinence nominale », voir la section subanta ci-après) et les tiN-anta « verbes » (litt. « ce qui se termine par une désinence verbale », voir la section tinanta ci-après), et détaille leurs unités constitutives respectives. L'ensemble constitue une structure hiérarchique, au sein de laquelle 32 classes d'unités se distribuent sur sept niveaux différents. On peut la représenter selon le schéma suivant :

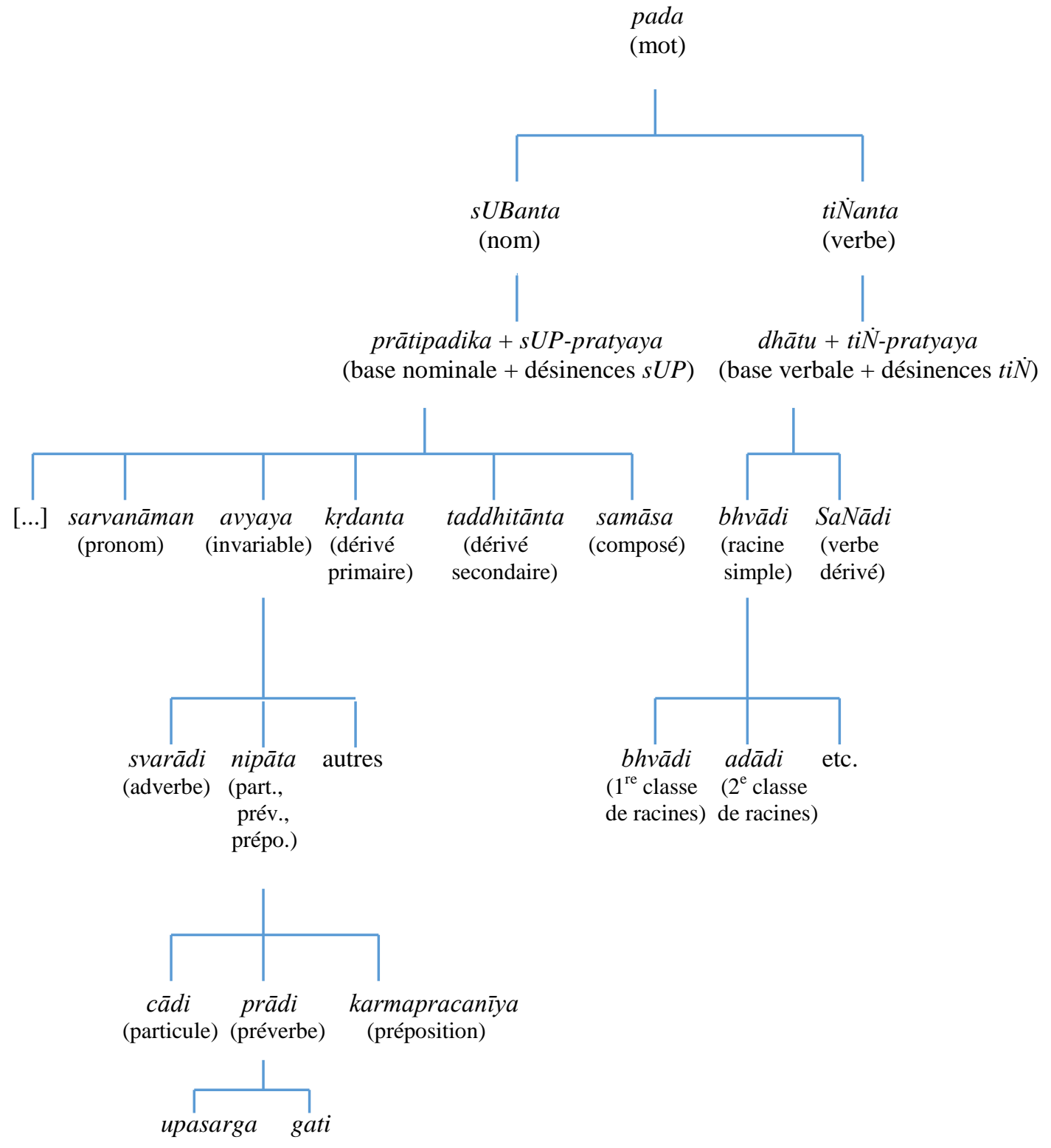

\footnotetext{
${ }^{7}$ Son traité, constitué approximativement de 4000 sùtra, permet la formation des mots (voir la section pada ciaprès) corrects d'une variété linguistique (correspondant grosso modo à ce que nous appelons aujourd'hui le « sanskrit classique ») dont il était probablement locuteur. La description entreprise par Pāninini s'expliquerait par une transformation profonde du paysage socio-religieux de son époque : les institutions mises en place par les Ārya sont concurrencées par des courants gagnant en popularité, tels le bouddhisme et le jä̈nisme, qui remettent en cause certains des fondements de la société brahmanique. Ces courants mettent également en question la suprématie de la langue sanskrite, cette dernière n'étant pas la langue de prêche de leur fondateur (le Bouddha aurait recouru à diverses variétés linguistiques, Mahāvīra - fondateur du jaïnisme - aurait employé le prakrit ardhamāgadhī).
} 
Le traité pāṇinéen ne semble pas avoir été composé pour être lu du début jusqu'à la fin : il n'y a ni progression ni continuité dans les faits décrits (cf. Pinault 1989a, p. 363). Très fréquemment, des sūtra qui doivent s'appliquer conjointement figurent dans des sections séparées par plusieurs sūtra, voire par plusieurs sections. Par conséquent, les opérations (ou accidents) qui concernent une classe ne succèdent pas systématiquement à la définition de la classe. Cette organisation s'explique par le fait que l'ordre d'application des sütra est moins gouverné par les faits de langue que par :

1) le type d'opération qu'enseignent les sūtra (opération générale vs particulière utsargalapavāda -, opération nécessaire $v s$ non-nécessaire - nityalanitya -, opération

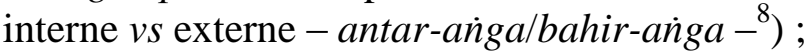

2) le fait que l'application de tel sūtra doit précéder l'application de tel autre ;

3) le fait d'éviter les répétitions dans la formulation des sūtra.

Quoi qu'il en soit, le noyau dur de la grammaire pāṇinéenne consiste en la dérivation des pada (toujours appréhendés dans un contexte phrastique), thème traité dans les $3^{\mathrm{e}}, 4^{\mathrm{e}}$ et $5^{\mathrm{e}}$ sections, les plus cohérentes du point de vue de l'ordre d'application des sūtra'

La majorité des grammaires post-pāṇinéennes (de tradition pāṇinéenne ou non) organisent - de manière plus ou moins apparente - les sütra en fonction des faits de langue ; ces grammaires suivent donc un plan thématique (elles sont souvent qualifiées de «topicwise grammars ${ }^{10}$ ). C'est dans les sphères bouddhistes et jaïnes, donc hors de l'école pāṇinéenne, que cet arrangement thématique émergea. En effet, lorsque les lettrés bouddhistes et jaïns décidèrent de composer leurs textes canoniques en sanskrit, le besoin d'avoir à leur disposition une grammaire moins ésotérique que celle de Pānini et plus adaptée aux besoins d'une audience non brahmanique se fit rapidement sentir. Ces grammaires à plan thématique sont principalement organisées par types de pada: les sūtra qui introduisent les unités constitutives d'un type de pada, ainsi que les sütra qui enseignent les opérations s'appliquant à l'intérieur des frontières de ces types de pada sont regroupées en une seule et même section. Adoptée par plusieurs grammaires rattachées à différentes écoles ${ }^{11}$, cette organisation thématique se développa au fil des siècles, gagnant progressivement en popularité (cf. Bali 1976, p. 38). L'ouvrage le plus célèbre du genre est sans conteste la Siddhāntakaumudī de Bhațtoji Dīkșita, grammaire de l'école pāṇinéenne composée vers la fin du XVI ${ }^{\mathrm{e}} \mathrm{s}$. - début du $\mathrm{XVII}^{\mathrm{e}}$ s. Cette nouvelle organisation des grammaires n'a pas eu de conséquences sur le système pāṇinéen des classes d'unités, dans la mesure où les grammaires à plan thématique préservent, dans l'ensemble, les définitions pāṇinéennes des classes, véritables pierres

\footnotetext{
${ }^{8}$ Les sūtra antar-anga enseignent des opérations dont les conditions (añga) sont internes (antar), alors que les sütra bahir-aìga enseignent des opérations dont les conditions (anga) sont externes (bahir). Lorsqu'un sūtra antar-angga et un sūtra bahir-anga interviennent dans la formation d'un mot, le premier s'applique avant le second. Ce principe, identique à celui de la parenthétisation (ou bracketing) de la linguistique occidentale moderne, est un mécanisme essentiel dans le système pāninéen car la démarcation des frontières entre pada se fonde entièrement sur lui (les opérations internes s'appliquent à l'intérieur des frontières entre pada, alors que les opérations externes s'appliquent à l'extérieur de ces frontières).

${ }^{9}$ La dérivation (dont le but ultime est la phrase) est très clairement le cœur du système pāṇinéen. Les sections 1 et 2, qui introduisent les notions fondamentales, la plupart des classes d'unités et des procédés techniques, peuvent être considérées comme préparant la dérivation. Les sections 6,7 et 8 présentent les résultats de la dérivation, les pada (pris comme constituants phrastiques), et permettent le traitement des phénomènes de jonction interne et d'accentuation (cf. Pinault 1989b, p. 371).

${ }^{10}$ Le terme sanskrit qui désigne (au sein de l'école pāṇinéenne, tout au moins) ces grammaires est prakriyā (littéralement «production, formation »). L'idée est que ces grammaires mettent l'accent sur la dérivation des mots et non sur les règles générales et les règles particulières qui les entravent (ce que fait notamment l'A).

${ }^{11}$ Le plus ancien ouvrage organisé thématiquement qui nous soit parvenu est le Kātantra de Śarvavarman, un lettré bouddhiste qui vécut probablement au $\mathrm{IV}^{\mathrm{e}} \mathrm{s}$.
} 
angulaires de la hiérarchie du système. De fait, le schéma des classes d'unités établi pour le traité pāṇinéen peut tout aussi bien représenter le système des classes d'unités des autres grammaires ; c'est le cas pour la Siddhāntakaumudī, entre autres ${ }^{12}$.

\section{Pada $\ll$ mot »}

Deux termes sanskrits, śabda- et pada-, peuvent être traduits par «mot». Ils sont employés dans des contextes différents.

śabda- (masc.) n'est pas un terme de la métalangue des grammairiens du sanskrit. Comme le rappelle Pinault (1992, p. 162), «[...] son sens fondamental est 'forme, expression' ; en védique, śabda désigne encore un simple 'bruit', ou un 'son' émis par un instrument de musique, par la gorge de l'animal ou de l'homme. Il s'agit probablement d'un dérivé à forme populaire et expressive de la racine śap- 'faire du bruit', pour les humains 'donner de la voix', d'où sa fixation au sens de 'maudire, jurer, lancer une imprécation', dès le Rgveda (cf. Mayrhofer, KEWAi. III, 296). » On le trouve néanmoins dans les textes grammaticaux, où il peut désigner tout aussi bien une phrase, un mot, une partie de mot, une syllabe, qu'un phonème.

pada- (nt.), en revanche, appartient proprement à la terminologie grammaticale sanskrite. Ainsi que le note Pinault (1992, p. 163) : «Originellement, padá- [dérivé du nom racine $p \bar{a} d-/ p a ́ d-$ 'pied'] désigne en védique le 'lieu où l'on marche' [...] d'où 'séjour, lieu' (volontiers secret) et 'trace' du pied ; c'est ce dernier emploi, qui comporte l'idée d'un signe à interpréter, que dérive le sens de 'mot', fixé à partir des Brāhmaṇas (commentaires sur le rituel), mais déjà acquis dans plusieurs passages des hymnes. Le point de départ de ce terme est mystique : le pada, caché dans la continuité de la parole, n'est accessible qu'aux savants et aux initiés, et donne un supplément de savoir. Pour les grammairiens aussi, il sera le produit d'une disquisition, dont le profane, le locuteur natif, n'a pas la notion ».

Contrairement à d'autres traditions grammaticales (la tradition grecque notamment, $c f$. Lallot 1983, §1.1), ce n'est pas en lien avec l'écriture que l'unité pada fut identifiée, mais avec la transmission orale des Veda. Les textes védiques ainsi que les disciplines d'érudition qui leur sont associées (les Vedānga) furent transmis oralement de maître à disciple, sur des centaines de générations. Ce mode de transmission a conduit très tôt à la mise au point de techniques élaborées de récitation pour garantir une mémorisation et une restitution parfaites de ces textes. La plus ancienne de ces techniques, la «récitation par mots » (padapātha), constitue la plus ancienne trace que nous ayons d'une segmentation de la chaîne parlée dans l'Inde ancienne. Le principe est le suivant : dans le texte «récité en continuité » (samhitāpātha), les règles euphoniques de jonction (sandhi) s'appliquent à l'intérieur des unités métriques (strophes et demi-strophes) récitées sans interruption ; dans le texte "récité par mots », certains sandhis sont abrogés et remplacés par des pauses, les unités délimitées par ces pauses, les pada, se présentent alors sous la forme qu'elles ont en finale absolue (cf. Pinault 2000, p. 223). Les pauses sont généralement faites là où les altérations phonétiques, que d'aucuns - occidentaux ${ }^{13}$ - nomment « sandhis externes » se réalisent, ce qui conduit à la délimitation des unités suivantes : mots fléchis, particules, thèmes (simples ou composés) qui précèdent une désinence casuelle ou un suffixe secondaire à initiale consonantique ${ }^{14}$, unités précédant les suffixes de dénominatif, unités précédant le suffixe de participe parfait -vāmss,

\footnotetext{
${ }^{12}$ Pour plus de détails, $c f$. Aussant (2016).

${ }^{13}$ Les théoriciens du langage de l'Inde ancienne ne distinguent pas des types de sandhis mais des types de bases suivies de certains suffixes. Autrement dit, lorsqu'il s'agit de décrire une opération phonétique, ce sont plus les conditions ou l'environnement de l'opération qui sont pris en compte que l'opération elle-même.

${ }^{14}$ Excepté les thèmes en $-t$ et $-s$ devant un suffixe signifiant « posséder ».
} 
constituants de composé qui précèdent le constituant venant en dernier dans la dérivation. L'ensemble de ces unités constitue la classe des pada, selon les auteurs des versions padapātha des textes védiques.

L'analyse que ces mêmes auteurs font des composés met en lumière deux autres caractéristiques des pada. Tout d'abord, comme l'a déjà noté Cardona (2014, p. 91-92), la pause est faite seulement au niveau de la dernière jonction du processus de dérivation, y compris lorsqu'il n'y a pas d'altération phonétique, comme dans prajṓ-patih̆ (ṚV 4.53.2 «maître (-patihh) des créatures (prajá́-)», constitué de trois unités pra-, jā- et pati-). Cela implique que les auteurs des versions padapātha concevaient les pada comme des unités syntaxiques, c'est-à-dire comme des séquences se terminant par une désinence nominale ou verbale ou comme des séquences s'étant terminées, à une étape du processus dérivationnel, par une telle désinence ${ }^{15}$. Ceci est confirmé par le fait que si le premier membre d'un composé ne se rencontre pas isolément (donc à l'état fléchi) dans le Rgveda (ci-après RV), il n'est pas analysé, i.e. séparé du second membre ${ }^{16}$. C'est donc sur la base de traits phonologiques et dérivationnels que les pada sont identifiés dans les versions padapātha des textes védiques. Ensuite, comme l'a montré Abhyankar (1974, p. 10), les pauses sont faites dans les composés lorsque le sens de chacun des constituants est mobilisé. Ainsi, les composés fonctionnant comme noms propres, tels que viśvámmitra (ṚV 3.53.9), ne sont pas analysés car alors le sens du composé serait «[le sage nommé] Viśvắmitra », non «ami de tout ». Les auteurs de versions padapātha des textes védiques considèrent donc les pada comme des unités signifiantes.

Pāninini, qui mentionne Sākalya - l'auteur de la version padapātha du Rgveda -, hérite de cette notion de pada. Elle apparaît de manière explicite dans les quatre sütra de l'Aștāadhyāȳ qui constituent la définition pānininéenne de la classe des pada. Le premier sūtra concerne les mots fléchis, alors que les trois suivants font référence, à quelques exceptions près, aux autres unités identifiées par Śākalya :

- sUP-tiNं-antam padam \| A 1.4.14 «Une unité qui se termine par une désinence nominale ou verbale [est appelée] pada»

- naḥ Kye || A 1.4.15 «[Une unité terminée par] - $n$ [est appelée pada] devant [un affixe] Kya (affixe de dénominatif) [en sorte que ledit $-n$ s'amuit selon A 8.2.7] ${ }^{17}$

- S-iti ca \| A 1.4.16 «[Une unité située] devant un suffixe à marqueur $S$ est également [appelée pada] ${ }^{18}$

\footnotetext{
${ }^{15}$ Les dérivés secondaires, qui sont formés à partir d'une séquence comprenant des formes fléchies sont analysés selon le même principe (ex. gó-mān (RV 4.2.5), qui est conçu comme dérivé de gāvo_asya santi « lui à qui appartiennent des vaches »).

${ }_{16}$ Notons que les auteurs des versions padapātha des textes védiques recourent fréquemment au critère de l'usage à l'état «isolé » pour déterminer le caractère signifiant d'une unité (cf. Jha 1992, p. 173 et 186). Il semblerait en revanche qu'ils n'aient pas fait usage de la méthode d'analyse fondée sur le raisonnement par anvaya-vyatireka «[concomitance] de présence et d'absence» (cf. Cardona 1967-68). Cette méthode, mentionnée par les grammairiens à partir de Kātyāyana ( $\mathrm{III}^{\mathrm{e}} \mathrm{s}$. av. JC), sera utilisée pour déterminer le caractère signifiant des deux types d'unités constitutives des pada (i.e. la base (prakrti) et le suffixe (pratyaya), unités qui ne se rencontrent pas à l'état isolé) et attribuer un sens propre à ces unités. L'analyse qu'elle implique est plus abstraite (elle porte sur les unités de première articulation) que celle fondée sur le critère de l'usage à l'état isolé. ${ }^{17}$ rājan- « roi » appartient à la classe des pada pour former rājīyati « il agit en roi » (avec amuïssement de - $n$ selon na-lopah prātipadika-antasya \| A 8.2.7 « Il y a amuïssement du - $n$ à la fin d'une base nominale [nommée pada, cf. A 8.1.16] »).

${ }^{18}$ bhavat- « votre » appartient à la classe des pada pour former, avec le suffixe chaS (-ìya), bhavadīyam «qui est vôtre » (avec substitution de - $d$ à $-t$ selon jhaLām jaŚo_ante \| A 8.2.39 «Les consonnes autres que les semivoyelles et les nasales (jhaLām) sont remplacées par des occlusives sonores non-aspirées (jaŚo) en finale [de pada] (ante) »). Quatre suffixes (tous secondaires) portent le marqueur $S$. Toutes les bases nominales construites
} 
- sV-ädiṣv a-sarvanāmasthāne $\|$ A 1.4 .17 « [Une unité située] devant la désinence $s U$ et les autres affixes [énumérés de A 4.1.2 à A 5.4.151, autrement dit, devant l'ensemble des désinences casuelles, suffixes du féminin et suffixes secondaires, est appelée pada], excepté s'il s'agit d'une désinence de cas forts sarvanāmasthāna) ${ }^{19}$

Pāninini inclut aussi les invariables dans la classe des pada et considère, de manière plus ou moins explicite (voir la section subanta ci-après), le sens comme un trait caractéristique des pada. L'analyse pāṇinéenne et celle des auteurs des versions padapāṭa se distinguent néanmoins sur plusieurs points. Deux doivent être mentionnés ici. Tout d'abord, l'Așțāahyāȳ vise à former des mots (des pada au sens de A 1.4.14) - conçus comme des constituants phrastiques - au moyen d'affixes (pratyaya). Ces affixes sont, selon certaines conditions sémantiques et contextuelles, combinés à des bases qui peuvent être des racines verbales (dhātu), des thèmes nominaux ou des pada (au sens de A 1.4.15-17). Autrement dit, dans l'analyse pāṇinéenne, le concept opératoire n'est pas pada (qui désigne le «produit fini » auquel doit aboutir le processus génératif) mais la séquence «base + affixe » (cf. Cardona 2012, p. 51). Les deux analyses divergent également du point de vue de leur «direction». Dans le contexte indien, on considère traditionnellement que la version padapātha d'un texte védique constitue la source de sa version samhitāpātha (cf. Cardona 1997b, p. 37). Cette conception, comme le note Renou (1960, p. 1), «tourne le dos à l'évolution historique » : les auteurs des versions padapātha sont nécessairement partis de la version saṃitāpātha pour élaborer la version padapātha des textes védiques (autrement dit, leur analyse part des phrases pour aller vers les constituants phrastiques, les pada). L'analyse grammaticale procède en sens inverse : les unités de départ sont les bases et les affixes, c'est-à-dire les unités constitutives des pada, et la grammaire génère la production de pada et de phrases $(v \bar{a} k y a)$. L'analyse qui fonde la version padapātha des textes védiques et l'analyse pāṇinéenne constituent donc deux approches fondamentalement distinctes : 1) d'un côté, une approche « déconstructive » (ou, dans la métalangue de la linguistique contemporaine, bottom up) qui vise à identifier les unités constitutives - et leurs modifications - des textes védiques dans le but d'assurer la correction de leur transmission ; 2) de l'autre, une approche «constructive » (ou top down) qui vise à générer des unités de plus en plus complexes jusqu'à la production de n'importe quelle phrase sanskrite correcte.

\section{Subanta «nom »}

Le terme subanta (écrit sUBanta dans la suite de l'article, les majuscules indiquant les marqueurs ${ }^{20}$ ) apparaît dans le sūtra A 1.4.14 sUP-tiNं-antam padam \| «Une unité qui se termine par une désinence nominale $(s U P)$ ou verbale $(t i \dot{N})$ [est appelée] pada ['mot'] ${ }^{21}$. Il n'est pas défini par ailleurs. $s U P$ - est l'abréviation $(s U . . . P)$ de la liste des désinences nominales donnée en A 4.1.2 :

\footnotetext{
avec ces suffixes portent le nom de pada, autrement dit, sont soumises aux opérations que l'ensemble des unités pada subissent.

${ }^{19}$ șaș- « six » appartient à la classe des pada s'il précède la désinence d'instr. pl. -bhis pour former ṣaḍhis (avec la substitution de $-d$ à $-s$ selon A 8.2.39).

${ }^{20}$ En sanskrit, anubandha. Renou (1942, vol. 1) en donne la définition suivante : «'élément annexé, exposant, indice grammatical' : se dit de certains phonèmes [...] qui, gén. en postposition, s'attachent à divers éléments grammaticaux, accréments, suffixes, substituts, thèmes et racines, afin d'en préciser les modalités d'emploi et not. de fournir des indications sur le ton, le degré vocalique, le mode de flexion ou de dérivation. Ces phonèmes sont par eux-mêmes dénués de valeur et tombent au cours de la formation du mot selon A 1.3.9. »

${ }^{21}$ Le $P$ de $s U P$ se sonorise au contact de sonores, d'où la forme $B$ dans $s U B$-anta.
} 
sU-au-Jas-am-auṬ-Śas-Ṭā-bhyām-bhis- $\dot{N} e-b h y \bar{a} m-b h y a s-\dot{N} a s I-b h y \bar{a} m-b h y a s-\dot{N} a s-o s-$ $\bar{a} m-\dot{N} i$-os-suP $\|$

«[Les désinences nominales] sU-au-Jas (nominatif sg., du., pl.), am-auT-Śas (accusatif sg., du., pl.), Țā-bhyām-bhis (instr. sg., du., pl.), Ne-bhyām-bhyas (datif sg., du., pl.), NasI-bhyām-bhyas (ablatif sg., du., pl.), Nas-os-ām (génitif sg., du., pl.), $\dot{N} i-$ os-suP (locatif sg., du., pl.) [sont employées après une unité se terminant par un suffixe $\dot{N} \bar{l}^{22}$ ou $\bar{a} P^{23}$ (suffixes de féminin) ou après un thème nominal]. »

Dans l'Aștâadhyāỹ de Pāninini, le nom (comme le verbe) est donc conçu sous un angle purement formel.

Les deux types de pada «mot», sUBanta «nom » et tiNanta «verbe » (voir section afférente ci-après), s'analysent en une base (prakrti) ${ }^{24}$ associée à un ou plusieurs suffixes

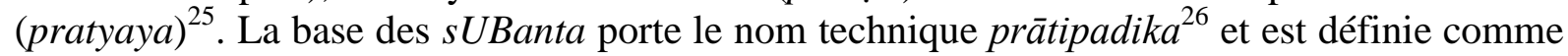
suit : arthavad adhātur apratyayah prātipadikam \| A 1.2.45 «[Une forme qui par elle-même] est pourvue d'un sens et qui n'est ni une racine verbale ni un affixe [est appelée] prātipadika ('base nominale') » ${ }^{27}$. Ce sūtra est complété par A 1.2.46 krt-taddhita-samāsāśs ca \| «Un dérivé primaire, un dérivé secondaire, un composé aussi [sont appelés] prātipadika ».

Cette base nominale (prätipadika) peut être de plusieurs sortes :

- il peut s'agir d'un pronom (sarvanāman) ${ }^{28}$, défini en A 1.1.27 sarva-ādīni sarvanāmāni $\|$ «[Les unités du groupe sarva ('tout') [sont appelées] sarvanāman »; ce groupe se compose de 29 unités $^{29}$ (pronoms personnels, démonstratifs, relatifs et interrogatifs, numéraux, principalement) ayant en commun des traits de flexion ;

- d'un dérivé primaire $(k r d a n t a)^{30}$; ces unités ne sont pas introduites telles quelles dans la grammaire de Pāṇini : celui-ci pose d'abord la classe des suffixes (pratyaya) en A 3.1.1-2, dont il traite dans trois sections (livres 3, 4, 5) sur six. Au sein de ces sections, de $\mathrm{A} 3.1 .93^{31}$ à A 3.4.117, il énumère et détaille les conditions d'emploi des suffixes primaires $(k r t)$, qui permettent la formation de dérivés tels que kartr« agent » (directement dérivé de la racine $k r$ - «agir, faire »);

- d'un dérivé secondaire (taddhitānta) ${ }^{32}$; tout comme les dérivés primaires, les dérivés secondaires ne sont pas introduits tels quels dans l'Aștâadhyāyzi , ce sont les conditions d'emploi des suffixes secondaires (taddhita) qui sont énumérées et détaillées de A

\footnotetext{
${ }^{22}$ Ex. kartrī- « celle qui fait », en regard de kartr- « celui qui fait ».

${ }^{23}$ Ex. ajā- « chèvre », en regard de aja- « bouc ».

${ }^{24}$ Terme non défini par Pāṇini.

${ }^{25}$ Littéralement, «ce qui vient contre ». Le terme est introduit par A 3.1.1 pratyayah «[Le nom technique] pratyaya ('affixe') [s'applique à tous les sütra qui suivent jusqu'à la fin du livre 5] » et A 3.1.2 paraś ca «Et [ledit affixe prend place] après [la racine verbale ou le thème nominal ». Notons que les désinences nominales et verbales sont aussi désignées au moyen du terme technique vibhakti (litt. «division ») : vibhaktiś ca \| A 1.4.104 « [Les séries de désinences nominales et verbales portent] aussi [le nom de] vibhakti ('désinence'). »

${ }^{26}$ Dérivé de pratipada- (« relatif à chaque mot ») signifiant « appartenant à chaque mot ».

${ }^{27}$ Cette formulation s'explique par le fait que, contrairement aux racines verbales et aux affixes, les prātipadika ne sont pas en nombre fini.

${ }^{28}$ Littéralement, « nom pour tout ».

${ }^{29}$ D'après l'éd. de Böhtlingk.

${ }^{30}$ Littéralement, «qui se termine par un suffixe krt (suffixe primaire) ». krtt- «qui fait », est un exemple de la formation.

${ }^{31} k r d$-ati $\dot{N} \|$ « [Jusqu’à A 3.4.117, tout affixe] excepté une désinence verbale [est appelé] krt. »

${ }^{32}$ Littéralement, « qui se termine par un suffixe taddhita (suffixe secondaire) »; taddhita- «bon pour $\mathrm{x} »$, est un exemple de valeur commandant l'emploi d'un suffixe secondaire. Par ex., à partir de la séquence ātmane hitam «bon pour soi », on peut former, à l'aide du suffixe -ìna, le dérivé àtmanīnam.
} 
4.1.76 à A 5.4.160, suffixes qui permettent la formation de dérivés tels que kartrtva«propriété d'être un agent » (dérivé du thème nominal (et dérivé primaire) kartr-) ;

- d'un composé (samāsa ${ }^{34}$ ) ; le terme est introduit en A 2.1.3 prāk kaḍāāt samāsah \| «[Depuis le sütra suivant] jusqu'à A 2.2.38 [toutes les formations décrites sont appelées] samāsa- ('composé') »; Pāninini distingue quatre classes de composés : invariabilisé (avyayībhāva- ${ }^{35}$ ), déterminatif (tatpurușa- ${ }^{36}$ ), possessif (bahuvrīhi- ${ }^{37}$ ) et copulatif (dvandva- $\left.{ }^{38}\right)$;

- d'un invariable (voir la section avyaya ci-après) ; le sūtra A $2.4 .82^{39}$ stipule l'amuïssement des désinences nominales après ce type d'unités ;

- de toute base qui, d'après Pāṇini, ne peut être dérivée (ex. vṛkṣa- «arbre ») ; il s'agit d'unités signifiantes qui ne sont ni des racines verbales (dhātu), ni des suffixes (pratyaya), ni des pronoms (sarvanāman), ni des invariables (avyaya), ni des dérivés primaires (krdanta), ni des dérivés secondaires (taddhitānta), ni des composés (samāsa). Pāninini ne donne pas de nom - autre que prātipadika - à ce type d'unités (elles sont représentées par [...] dans le schéma).

\section{Tinanta « verbe »}

Le terme tinanta (écrit tiNanta dans la suite de l'article, les majuscules indiquant les marqueurs ${ }^{40}$ ) n'apparaît que dans le sūtra A 1.4.14 sUP-tiN-antam padam \| «Une unité qui se termine par une désinence nominale $(s U P)$ ou verbale $(t i \dot{N})$ [est appelée] pada ['mot']. » $t i \dot{N}$ - est l'abréviation $(t i \ldots \dot{N})$ de la liste des désinences verbales données en A 3.4.78:

tiP-tas-jhi-siP-thas-tha-miP-vas-mas-ta-ätām-jha-thās-āthām-dhvam-iṬ-vahi-mahi $\dot{N} \|$ «[Les désinences verbales] tiP-tas-jhi ( $3^{\mathrm{e}}$ pers., actif, sg., du., pl.), siP-thas-tha $\left(2^{\mathrm{e}}\right.$ pers., actif, sg., du., pl.), miP-vas-mas ( $1^{\mathrm{re}}$ pers., actif, sg., du., pl.), ta-ātām-jha $\left(3^{\mathrm{e}}\right.$ pers., moyen, sg., du., pl.), thās-āthām-dhvam ( $2^{\mathrm{e}}$ pers., moyen, sg., du., pl.), iT-vahi$\operatorname{mahiN}\left(1^{\text {re }}\right.$ pers., moyen, sg., du., pl.) [se substituent à $L$ (terme métalinguistique générique pour les affixes modo-temporels)]. »

Le verbe, comme le nom, est donc conçu sous un angle purement formel ${ }^{41}$.

\footnotetext{
${ }^{33}$ taddhitāh $\|$ «Les suffixes secondaires [sont l'objet des sūtra qui suivent jusqu'à la fin du livre 5]. »

${ }^{34}$ Littéralement, « réunion ».

${ }^{35}$ Littéralement, « accession à l'état d'invariant », composé adverbial. Pāṇini classe ces unités dans la catégorie des avyaya (voir la section afférente ci-après).

${ }^{36}$ « Le serviteur de $\mathrm{x} »$, ex. de la formation.

37 «Qui a beaucoup de riz », ex. de la formation.

${ }^{38}$ Littéralement, « paire ».

${ }^{39}$ avyayād $\bar{a} P-s U P a h \|$ «[Il y a amuïssement du suffixe général du féminin $\bar{a} P$ et des désinences nominales après un invariable. »

${ }^{40}$ Voir note 20.

${ }^{41}$ La question du sens des verbes sera discutée par les commentateurs de Pāṇini. Kātyāyana (III ${ }^{\mathrm{e}}$ s. av. JC) considère que le sūtra A 1.3.1 pose deux problèmes : 1) si dhātu-est seulement défini par extension, il peut s'appliquer aux homonymes des racines verbales (ex. $y \bar{a}$ - «aller » et $y \bar{a}$ - pronom relatif féminin ; 2) dans le Dhātupātha, les racines verbales d'un groupe sont récitées en continu (bhvédha... et non bhū-, edha-,...) et rien n'est dit au sujet de la délimitation de ces unités. Patañjali ( $\mathrm{II}^{\mathrm{e}} \mathrm{s}$. av. JC) proposera une première définition pour résoudre ces problèmes, puis une deuxième... pour aboutir à une définition faisant intervenir les actants : un dhātu exprime une action $(k r i y \bar{a})$, cette action étant à concevoir comme la façon dont les actants participent à la réalisation d'un processus.
} 
Les verbes, tout comme les noms, s'analysent en une base (prakṛti) ${ }^{42}$ associée à un ou plusieurs suffixes (pratyaya) ${ }^{43}$. La base des verbes porte le nom technique $d h \bar{a} t u^{44}$ et est définie comme suit : bhūv-ädayo dhātavah $\|$ A 1.3.1 « [Les unités du groupe] bhü- ('devenir') [sont appelées] dhātu», sūtra complété par A 3.1.32 SaN-ādy-antā dhātavah \| "[Les formations terminées par] l'affixe $S a N$, etc. [sont appelées] dhātu ». Il y a donc deux classes de $d h \bar{a} t u$.

$b h \bar{u}$ - est la toute première racine verbale du tout premier groupe listé dans le Dhātupātha, le catalogue des verbes utilisé par Pāṇini. Ce catalogue comprend près de 2000 racines verbales réparties en dix classes (gana), désignée d'après le premier élément de chaque classe. Ces différentes classes se répartissent selon la formation du présent et subsidiairement selon d'autres critères (voix, accentuation), comme suit :

- $b h \bar{u}$-, etc. : racines à présent thématique sans affixe à degré plein (ex. ${ }^{*} b h o-a-t i=$ bhav-a-ti «il/elle devient »)

- $a d-$, etc. : racines à présent athématique radical (ex. at-ti «il/elle mange »)

- juhoti-, etc. : racines à présent athématique à redoublement (ex. ju-ho-ti «il/elle fait une oblation », racine $h u-$ )

- div-, etc. : racines à présent thématique à affixe $y$ (ex. $d \bar{\imath} v-y-a-t i$ «il/elle joue »)

- su-, etc. : racines à présent athématique à affixe nu/no (ex. su-no-ti «il/elle pressure $\gg)$

- tud-, etc. : racines à présent thématique sans affixe à degré réduit (ex. tud-a-ti «il/elle fait mal »)

- rudh-, etc. : racines à présent athématique à affixe $n / n a\left(\right.$ ex. ${ }^{*} r u-n a-d h-t i=r u-n a-$ $d d h i$ «il/elle investit »)

- tan-, etc. : racines en - $n$ à présent athématique à affixe $u / o$ (ex. tan-o-ti «il/elle étend $\gg)$

- $k r \bar{l}-$, etc. : racines à présent athématique à affixe $n \bar{l} / n \bar{a}$ (ex. $k r \bar{\imath}-n a-t i$ «il/elle achète »)

- cur-, etc. : racines à présent thématique à affixe aya (ex. cor-aya-ti «il/elle vole»)

L'expression «bhu- etc. » (bhūv-ădi-) permet de référer à l'ensemble du catalogue des verbes, de manière particulièrement économique. Cet ensemble de racines constitue l'une des deux classes de dhātu.

L'autre classe de dhātu rassemble des formes terminées par certains affixes («SaN, etc. »), donc des formes verbales dérivées. Les affixes $S a N$ sont principalement employés pour former les désidératifs (ex. pipāsati «il/elle désire boire», sur pā- «boire»). Par « etc. », il faut entendre l'affixe yaj, utilisé pour former les intensifs (ex. rorūyate «il/elle pleure violemment », sur $r \bar{u}$ - «pleurer »), $N i C$, utilisé pour former les causatifs (ex. kārayati «il/elle fait faire », sur $k r-$ «faire ») et $K y a C$, utilisé pour former les dénominatifs (ex. vrșāyáte « il mugit comme un taureau », sur vrṣan- «taureau »).

Avyaya « invariant »

\footnotetext{
${ }^{42}$ Terme non défini par Pāninini.

${ }^{43}$ Voir note 25.

${ }^{44}$ Littéralement, « fondation, partie essentielle », dérivé de la racine $d h \bar{a}$ - «poser, établir ».
} 
La classe des avyaya ${ }^{45}$, les invariables, constitue une sous-catégorie de la classe des thèmes nominaux (prātipadika, voir section subanta). Leurs désinences nominales sont amuïes par l'application du sūtra A 2.4.82. Un principe pāṇinéen veut que la validité d'un fait, dont la cause est un élément qui s'amuït, demeure une fois ledit élément effectivement amuï ; dans le cas présent, la validité du nom technique sUBanta («nom ») demeure une fois les désinences nominales amuïes. Du fait de ce principe, les invariables sont des noms (sUBanta) et, $a$ fortiori, des mots (voir la section pada) ${ }^{46}$.

La classe des invariables est subdivisée en deux sous-catégories : la classe des svarādi et celle des nipāta.

La classe des svarādi («svah, etc. ») ${ }^{47}$ se compose d'unités telles que : adverbes de lieu (ex. antar « au milieu »), de manière (ex. śanais « doucement »), de circonstance (ex. yugapat « au même moment ») et de cause (ex. atha «ainsi $»)^{48}$. Cette classe $\left(\mathrm{n}^{\circ} 254 \mathrm{du}\right.$ Ganapạtha ${ }^{49}$ ) inclut 150 items (d'après l'édition de Böhtlingk).

La classe des nipāta ${ }^{50}$ rassemble des particules modales (interjections) ou conjonctives, des préverbes et des prépositions. Pāṇini la conçoit comme se subdivisant en trois sous-catégories : la classe des cādi, celle des prädi et celle des karmapravacanīya.

La classe des cādi («ca, etc. ») ${ }^{51}$ (n॰85 du Gaṇapātha) se compose principalement de particules exprimant une valeur conjonctive, émotionnelle ou interjective. Elle inclut 195 items (d'après l'édition de Böhtlingk).

La classe des prādi («pra, etc. ») ${ }^{52}\left(\mathrm{n}^{\circ} 154\right.$ du Gaṇapạtha) inclut 22 items (d'après l'édition de Böhtlingk). Ces items, lorsqu'ils sont directement liés à des verbes (i.e., lorsqu'ils fonctionnent comme préverbes), appartiennent à deux sous-classes, celle des upasarga $a^{53}$ et celle des gati $^{54}$. Les grammairiens ultérieurs à Pānini disent des upasarga et gati qu'ils

\footnotetext{
${ }^{45}$ Littéralement «qui ne change pas ». Le terme est introduit par le sūtra A 1.1.37 svar-ādi-nipātam avyayam \| «[Les mots du groupe] svah ('ciel (demeure des Dieux)') et les nipāta ('particule') [sont appelés] avyaya ('invariable') ».

${ }^{46}$ Notons cette remarque formulée par Chatterji (1964, p. 450) : «Pāninini evidently divides all parts of speech into 'subanta' and 'tinanta' ('vyayavant' of the older grammarians) and 'avyaya' ». Selon ce spécialiste reconnu du Vyākaraṇa, Pāninini, sur la base d'une distinction ancienne entre formes variables (vyayavant) et formes invariables (avyaya) (dont je n'ai pas trouvé trace), aurait distingué trois types de mots (pada). Cette affirmation, bien qu'elle contredise la définition pāṇinéenne de pada, est néanmoins intéressante en ce qu'elle permet d'établir un lien entre la classification pāṇinéenne des pada et celle, probablement plus ancienne, qui distingue quatre classes de pada : noms (nāman), verbes (ākhyāta), prépositions (upasarga) et particules (nipāta) (cf. supra le développement initial sur la tradition grammaticale sanskrite).

${ }^{47}$ Le terme est introduit en A 1.1.37, $c f$. note 45.

${ }^{48}$ Faddegon (1936, p. 10) emploie l'expression « descriptive adverbs » pour les désigner.

${ }^{49}$ Le Gaṇapātha («récitation des groupes ») se compose de 261 listes d'unités autres que les racines verbales, constituées principalement de thèmes nominaux, de pronoms et de numéraux, etc. et arrangées par ordre alphabétique. Certaines listes sont closes, tandis que d'autres, les ākrtigaṇa, sont à considérer comme des énumérations de spécimens.

${ }^{50}$ Littéralement, « qui tombe » sous-entendu « tout fait », i.e., sans processus formatif. Le terme est introduit par le sūtra A 1.4 .56 prāg rīśvarān nipātāh \| «[Depuis le sūtra suivant] jusqu'à 97 [les formes décrites sont appelées] nipāta ('particules') ».

${ }^{51}$ Le terme est introduit par le sūtra A 1.4.57 ca-ādayo_asattve \| « [Les mots du groupe] ca ('et') [sont appelés] nipāta ('particule') quand ils ne désignent pas une substance ». Ex. paśu- est particule lorsqu'il signifie « certes », non quand il exprime une substance, comme dans paśur vai puruṣah « L'homme est une victime ».

${ }^{52}$ Le terme est introduit par le sūtra A 1.4 .58 pra-ādayah $\|$ «[Les mots du groupe] pra [sont appelés nipāta ('particule') quand ils ne désignent pas une substance ».

${ }^{53}$ Littéralement, «qui est ajouté, joint, attaché ». Le terme est introduit par le sūtra A 1.4 .59 upasargāh kriyāyoge $\|$ « [Les mots du groupe pra portent le nom] upasarga ('préverbe') quand ils sont reliés à l'action ».

${ }^{54}$ Littéralement, «marche ». Le terme est introduit par le sūtra A 1.4.60 gatiś ca \| «[Les mots du groupe pra, quand ils sont reliés à l'action, portent] aussi [le nom de] gati ». La classe des gati ne se réduit pas aux mots du groupe pra.
} 
spécifient l'action (kriyā-viśeșaka). Lorsque les mots du groupe pra ne sont pas directement liés à des verbes, ils fonctionnent comme préfixes de déverbatifs ou d'indéclinables.

La classe des karmapravacaniyya ${ }^{55}$ rassemble des prépositions régissantes (11 unités) $^{56}$. Selon les grammairiens ultérieurs, les prépositions différencient (i.e., nuancent) une relation (sambandhasya bhedakah).

Si l'on étudie les sūtra relatifs à l'ensemble des unités avyaya, il apparaît que la grande majorité de ces unités est associée à un sens, mais rien n'est dit, dans l'Aștāadhyāȳi , de la manière dont ce sens est exprimé (i.e. directement ou indirectement). La question sera traitée après Pāninini ${ }^{57}$.

\title{
Émilie Aussant
}

CNRS/Université de Paris, Laboratoire d'Histoire des Théories Linguistiques

\begin{abstract}
Abréviations
A - Aștāâhyāyō de Pāṇini.

KEWAi. III - Kurzgefasstes etymologisches Wörterbuch des Altindischen (voir Mayrhofer). RQV - Rgveda.
\end{abstract}

\section{Références primaires}

Kātantra-Vyākaraṇa. Edited by R.S. Saini, Delhi - Varanasi, Bharatiya Vidya Prakashan, 1987.

The Nighantu and the Nirukta. Edited and translated by L. Sarup, Delhi, Motilal Banarsidass, 1998 ( $1^{\text {re }}$ ed. : Londres, 1920-27).

Pāṇini's Grammatik. Herausgegeben, übersetzt, erläutert und mit verschiedenen indices versehen von Otto Böhtlingk, Abteilung I \& II (en un volume), Delhi, Motilal Banarsidass (éd. indienne basée sur la $2^{\text {nde }}$ éd. : Leipzig, $1887 ; 1^{\text {re }}$ éd. : 1839-40).

The Siddhāntakaumudī of Bhattoji Dīkșita. Edited and translated into English by Śríśa Chandra Vasu, 2 vol., Delhi, Motilal Banarsidass, 1995 (1 ${ }^{\text {re }}$ éd. : Allahabad, 1906).

\section{Références secondaires}

ABHYANKAR, Kashinath Vasudev, 1961. A Dictionary of Sanskrit Grammar, Baroda, Oriental Institute.

\footnotetext{
55 Littéralement, «qui exprime, explique l'acte». Le terme est introduit par le sūtra A 1.4 .83 karmapravacanīyāh $\|$ « [Depuis le sūtra suivant jusqu'à 97, les particules qui vont être décrites portent le nom de] karmapravacanīya ('préposition')».

${ }^{56}$ Par ex., anu « en conséquence de »+ acc., « avec »+ instr., « après »+ acc., apa et pari « sauf »+ abl.

${ }^{5}$ À partir de Patañjali et jusqu'à Nāgeśa, les grammairiens défendent principalement l'idée selon laquelle les upasarga, les nipāta et les karmapravacanīya sont de simples «indicateurs » (dyotaka, qui, selon Nāgeśa, signifie vyañjaka « suggestif ») du sens et non de véritables porteurs de sens (vācaka, voir Abhyankar 1961, p. 81). Bhartrhari (VP 2.188) aborde le fonctionnement sémiologique des upasarga sous un angle différent : ces unités, selon lui, peuvent 1) être pourvues d'un sens propre et l'exprimer directement (vācaka) ; 2) être les indicateurs (dyotaka) d'un sens qui existe dans la racine verbale mais qui n'est pas exprimé par ladite racine ; 3) aider (sahakârr $\bar{l}$ ) la racine verbale à exprimer son sens (la racine et l'upasarga expriment le sens conjointement).
} 
ABHYANKAR, Kashinath Vasudev, 1974. Veda-padapātha-carcā, together with the text of Upalekhasūtram of Bhāradvāja Bṛhaspati edited with explanation in English, Poona, Bhandarkar Oriental Research Institute.

Aussant, Émilie, 2016. "Classifications of Words in Ancient Sanskrit Grammars », in History of Linguistics 2014: Selected papers from the 13th International Conference on the History of the Language Sciences (ICHoLS XIII), Vila Real, Portugal, 25-29 August 2014, ed. by Carlos Assunção, Gonçalo Fernandes \& Rolf Kemmler. Amsterdam \& Philadelphia, John Benjamins, Studies in the History of the Language Sciences 126, 97-109.

BALI, Suryakant, 1976. Bhaț̣oji Dīkșita. His Contribution to Sanskrit Grammar, Delhi, Munshiram Manoharlal.

CARDONA, George, 1967-68. «Anvaya and vyatireka in Indian grammar », The Adyar Library Bulletin 31-32, 313-352.

CARdona, George, 1997a [1988]. Pānini. His work and its traditions, volume I : Background and Introduction, $2^{\text {nd }}$ revised and completed edition, Delhi, Motilal Banarsidass.

CARDONA, George, 1997b. « Vedic Tradition and Descriptions of Grammarians », Witzel, M. (ed.), Inside the texts, beyond the texts - New approaches to the study of the Vedas (Proceedings of the International Vedic Workshop Harvard University, June 1989), Cambridge, Harvard University, 33-38.

CARdonA, George, 2012. «Pāṇini and Padakāras », Voegeli, F., Eltschinger, V., Feller, D., Candotti, M.P., Diaconescu, B. \& M.A. Kulkarni (ed.), Devadattīyam - Johannes Bronkhorst Felicitation Volume, Bern, Peter Lang, 39-61.

CARdonA, George, 2014. «Segmentation of Vedic texts : padapāthas », Bulletin d'Études Indiennes 32 (Actes du workshop « The Indian Traditions of Language Studies », ICHoLS XI, Potsdam, éd. par É. Aussant et J.-L. Chevillard), 87-100.

ChATterJI, Kshitish Chandra, 1964. Technical terms and technique of Sanskrit Grammar, Calcutta, University of Calcutta ( $1^{\text {re }}$ éd. : 1948).

FADDEGON, Barend, 1936. Studies on Pānini's grammar (= Verhandelingen der koninklijke Akademie van Wetenschappen te Amsterdam, Nieuwe Reeks, 38.1), Amsterdam, N.V. noordhollandsche Uitgeversmaatschappij.

JHA, Vashishtha Narayan, 1992. A Linguistic Analysis of the Rgveda-Padapattha (PrePāninian Grammatical Traditions, Part I), Delhi, Sri Satguru Publications.

LALLOT, Jean, 1983. "Les origines de la théorie grammaticale dans l'antiquité grecque », Travaux d'Histoire des Théories Linguistiques 1, 31-47.

MAYRHOFER, Manfred, 1956-1963-1976. Kurzgefasstes etymologisches Wörterbuch des Altindischen. I-III, Heidelberg, Bd. IV : Register (avec Heinz Dieter Pohl, Rüdiger Schmitt et Ronald Zwanziger), 1980. 
PINAult, Georges-Jean, 1989a. "Procédés pāṇinéens », Auroux, S. (éd.), Histoire des idées linguistiques, Tome 1, Liège, Pierre Mardaga, 354-370.

PINAUlt, Georges-Jean, 1989b. «Le système de Pāṇini », Auroux, S. (éd.), Histoire des idées linguistiques, Tome 1, Liège, Pierre Mardaga, 371-400.

PINAULT, Georges-Jean, 1992. «Le mot et l'analyse morphologique selon la grammaire indienne », Lalies 10, 159-176.

PINAUlT, Georges-Jean, 2000. «Śākalya » (notice 4301), Histoire Épistémologie Langage, Hors-Série 3 - Corpus représentatif des grammaires et des traditions linguistiques, tome 2, 223-226.

RENOU, Louis, 1942. Terminologie grammaticale du sanskrit, Paris, Bibliothèque de l'École des hautes études en sciences historiques et philologiques, Champion, 3 vol.

RENOU, Louis, 1960. «La forme et l'arrangement interne des Prātiśākhya », Journal asiatique 248, 1-40.

ThIEME, Paul, 1982-1983. «Meaning and form of the 'grammar' of Pāninini, Studien zur Indologie und Iranistik 8/9, 3-34. 